\title{
Aspectos do design de exposições de Frederick Kiesler
}

Marcelo Rafael de Carvalho é Mestrando em Design pela pós-graduação da Universidade Anhembi Morumbi. Bolsista Capes Prosup/UAM/Bolsa. Possui graduação em Comunicação Social pela Universidade Mackenzie. Atuou como supervisor do serviço educativo do MAB (Museu de Arte Brasileira), assistente de coordenação do educativo do Instituto Cultural Itaú e coordenador da ação educativa da $26^{\circ}$ Bienal de São Paulo.<marcelorafac@gmail.com> ORCID: 0000-0001-5134-5963
Resumo $O$ presente artigo tem como objetivo contribuir com os estudos do design de exposições e campos correlatos ao apresentar e organizar numa perspectiva histórica algumas das contribuições e propostas realizadas por Frederick Kiesler (1890-1965), designer de exposições, arquiteto, cenógrafo, artista e teórico, para espaços expositivos na primeira metade do século XX. Seus projetos expositivos desenvolvidos na Europa e nos Estados Unidos, privilegiavam estruturas adaptáveis e moveis, capaz de responder as percepções e aos movimentos do usuário, os quais estariam envolvidos de forma dinâmica nas proposições, sendo assim enfatizado o papel ativo do visitante.

Palavras chave Frederick Kiesler, Design de exposições, História das exposições. 
Mirtes Marins de Oliveira é Curadora, pesquisadora e docente da pós-graduação (mestrado e doutorado) em Design na Universidade Anhembi Morumbi. Pós-Doutorado em Educação - DE-USP. Possui graduação em Educação Artística- Artes Plásticas pela Universidade de São Paulo (1986), mestrado (1997) e doutorado (2002) em Educação: História, Política, Sociedade pela Pontifícia Universidade Católica de São Paulo. <mirtescmoliveira@gmail.com > ORCID: 0000-0002-7132-0875

\section{Aspects of Frederick Kiesler's design of exhibition}

Abstract The paper aims to contribute to the studies of exhibition design and related fields by presenting and organizing in a historical perspective some of the contributions and proposals made by Frederick Kiesler (1890-1965), exhibition designer, architect, set designer, artist and theorist, for exhibition spaces in the first half of the 20th century. His exhibition projects developed in Europe and the United States, favored adaptable and mobile structures, capable of responding to the user's perceptions and movements, which would be dynamically involved in the propositions, thus emphasizing the active role of the visitor.

Keywords Frederick Kiesler, Design of Exhibitions, Histories of Exhibitions.

\section{Aspectos del diseño de la exposición de Frederick Kiesler}

Resumen Este artículo tiene como objetivo contribuir a los estudios de diseño de exposiciones y campos relacionados presentando y organizando en una perspectiva histórica algunas de las contribuciones y propuestas hechas por Frederick Kiesler (1890-1965), diseñador de exposiciones, arquitecto, escenógrafo, artista y teórico, para espacios expositivos en la primera mitad del siglo XX. Sus proyectos de exhibición desarrollados en Europa y Estados Unidos, favorecieron estructuras adaptables y móviles, capaces de responder a las percepciones y movimientos del usuario, que estarían involucrados dinámicamente en las proposiciones, enfatizando así el papel activo del visitante.

Palabras clave Frederick Kiesler Diseño de exposiciones, historia de exposiciones. 


\section{Introdução}

É crescente o interesse e relevância que os estudos de design de exposições vêm adquirindo, não somente para o campo do design, mas também para campos correlatos, como o da história e crítica de arte e estudos culturais. $O$ presente artigo tem como objetivo contribuir com os estudos das exposições ao apresentar e organizar numa perspectiva histórica algumas das contribuições e propostas realizadas por Frederick Kiesler para espaços expositivos na primeira metade do século XX.

Frederick Kiesler foi arquiteto, cenógrafo, designer de exposições, artista e teórico. Seus trabalhos em Berlim, Viena e Paris como designer cenográfico e exposições no início da década de 1920 o colocam em evidência aproximando-o de membros do movimento Dada e De Stijl. Em 1926 muda-se para New York onde projetará um cinema, ambientes cênicos, exposições, vitrines, móveis e luminárias. Cofundador do International Theatre Arts Institute in Brooklyn, foi diretor cênico da Juilliard School of Music de 1934 a 1957 e conferencista e pesquisador visitante nas universidades de Columbia e Yale entre 1936 e 1952, nas quais criou um dos primeiros laboratórios de pesquisa em design a partir de um modelo inovador e experimental.

Portanto, o artigo será dividido em três partes. Em uma primeira será apresentado sua teoria na qual defendia existir uma relação integral entre cada objeto ou indivíduo e seus ambiente, e suas propostas expositivas desenvolvidos na década de 1920 na Europa. Na segunda parte será discorrido sobre sua produção em New York a partir de 1926 até a concepção da galeria The Art of This Century de Peggy Guggenheim. Na terceira serão descritas as duas exposições realizadas em 1947, resultado da aproximação e amadurecimento das relações entre Kiesler e o círculo de artistas surrealistas.

\section{Kiesler no Velho Mundo}

A abordagem de Kiesler desafiou e superou as fronteiras convencionais de várias disciplinas como arte, arquitetura, teatro e design, combinando em suas pesquisas os campos da estética, da biologia, da morfologia e da psicologia a fim de desenvolver novas práticas de construções espaciais.

Elaborou uma teoria denominada Correalismo, na qual defendia a crença numa relação integral entre cada objeto ou indivíduo e seu ambiente. Em suas palavras, correalismo representaria "a dinâmica da interação contínua entre o homem e seus ambientes naturais e tecnológicos" ${ }^{\text {(KIES- }}$ LER $^{2}, 1939$, p. 61 apud GOODMAN, 1989, p.63, tradução nossa).

Em seu Segundo Manifesto do Correalismo, declara: 
Fig 1. Display "T"

Fonte http://www.bildarchivaustria.

at/Preview/13535963.jpg
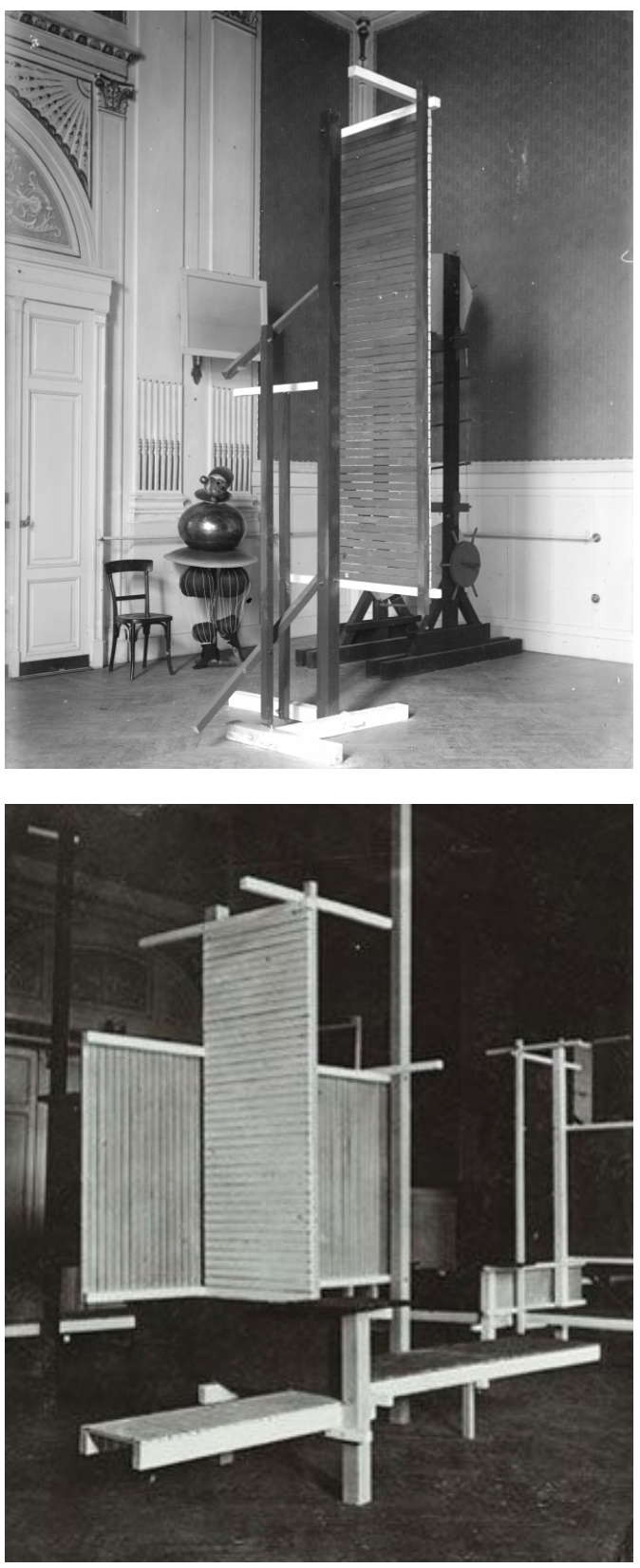

Fig 2. Combinação de display "T" e "L" Fonte https://za.pinterest.com/ pin/615867317765268879/
O objeto de arte tradicional, seja uma pintura, uma escultura ou uma peça de arquitetura, não é mais visto como uma entidade isolada, mas deve ser considerado dentro do contexto deste ambiente em expansão. 0 ambiente se torna igualmente tão importante quanto o objeto, se não mais, porque o objeto respira para o ambiente e também inspira as realidades do ambiente, independentemente do espaço, próximo ou distante, ao ar livre ou interno ${ }^{3}$ (KIESLER ${ }^{4}$, 1965, p. 6 apud STANISZEWSKI, 2001, p.8, tradução nossa)

Embora a proposição de Kiesler definia o homem integrado a um sistema de forças podendo ser interpretado como uma filosofia cósmica (PHILLIPS, 1989a) ou mística, seu conceito de correalismo é, como destacado por Staniszewski (2001), antiessencialista, pois a criação de significados origina-se no sujeito, culturalmente determinado no tempo e no espaço. Sua abordagem espacial enfatizava a participação ativa do espectador. Seus projetos vislumbravam uma estrutura adaptável, móvel, no qual o indivíduo estaria envolvido de forma dinâmica, em um ambiente adaptável. Uma arquitetura como Phillips (2017) denominou elástica, capaz de responder as percepções e aos movimentos do usuário.

Já em 1924, seu projeto para a Internationale Ausstellung neuer Theatertechnik (Exposição Internacional de Nova Técnica de Teatro) que integrava o Musik und Theaterfest der Stadt Wien (Festival de Música e Teatro da Cidade de Viena) indicava suas ideias.

Nessa exposição, segundo Staniszewski (2001), Kiesler reuniu trabalhos de artistas como El Lissitzky (1890-1941), Leon Bakst (1866-1924), Alexandra Exter (1882-1949), Natalia Goncharova (1881-1962), Fernand Léger (1881-1955), Francis Picabia (1879-1953), Enrico Prampolini (1894-1956), Hans Richter (1888-1976) e Oskar Schlemmer (1888-1943) numa construção espacial, com um novo método de design de exposição que chamou de Leger e Trager ou $L$ e $T$ (Figura 1e Figura 2). Um sistema flexível de exibição constituído por vigas verticais e horizontais que permitiam expor objetos bi e tridimensionais, podendo ser organizado de múltiplas maneiras e sustentar painéis tanto horizontalmente como verticalmente. Os trabalhos podiam ser apresentados de forma independente ou agrupados, ao visitante era permitido movimentar e ajustar partes da estrutura para apreciação das obras a seu bel-prazer.

$\mathrm{O}$ arranjo proposto por Kiesler havia retirado as obras das paredes, como geralmente são encontrados nas exposições convencionais. As transparências das aberturas na estrutura, possibilitavam vislumbrar um espaço integrado que evocava um sentido de continuidade (Figura 3 e Figura 4). Além disso, ao permitir a interatividade por parte do espectador, reconhecia-se a importância da percepção do indivíduo na construção de sentidos para as obras. 
Fig 3. Exposição Internacional de Nova Técnica de Teatro, Viena, 1924 (vista área)

Fonte https://www.kiesler.org/en/vienna-1924/pin/615867317765268879/

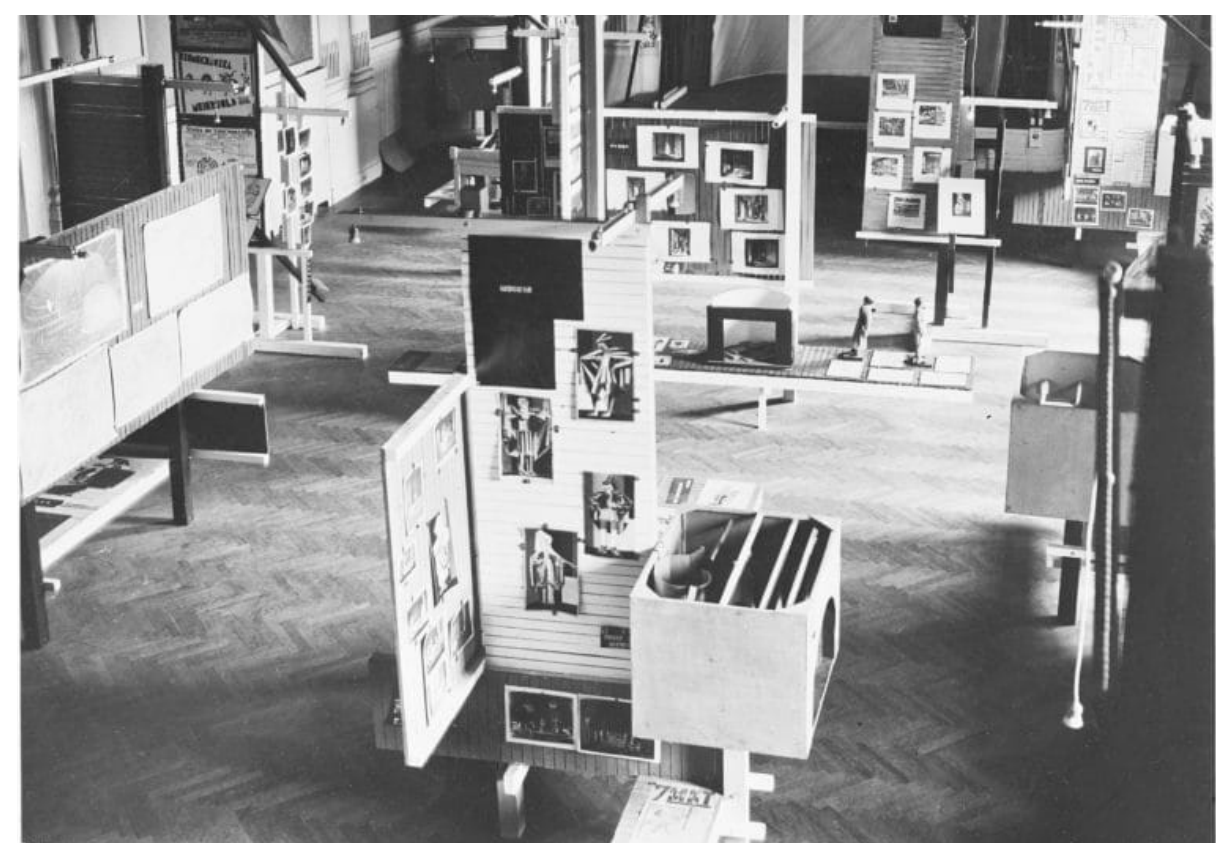

Como reconhece Staniszewski (2001), as "estruturas independentes de Kiesler trouxeram as obras de arte para o espaço do espectador e criaram o que ele chamou de 'transparência variada"': ${ }^{5}$ (p. 4, tradução nossa).

Cabe destacar tratar-se de unidades flexíveis, concebidas como arquitetura temporária, podendo facilmente serem adaptadas, incorporando lâmpadas elétricas e novas cores a partir das necessidades e configurações especificas dos novos espaços.

1924 FREDERICK KIESLER : “EXPOSITION DES NOUVELLES TECHNIQUES THÉATRALES » VIENNE

Fig 4. Exposição Internacional de Nova Técnica de Teatro, Viena, 1924

Fonte http://www.beaudouin-architectes.fr/2016/12/frederick-kiesler/

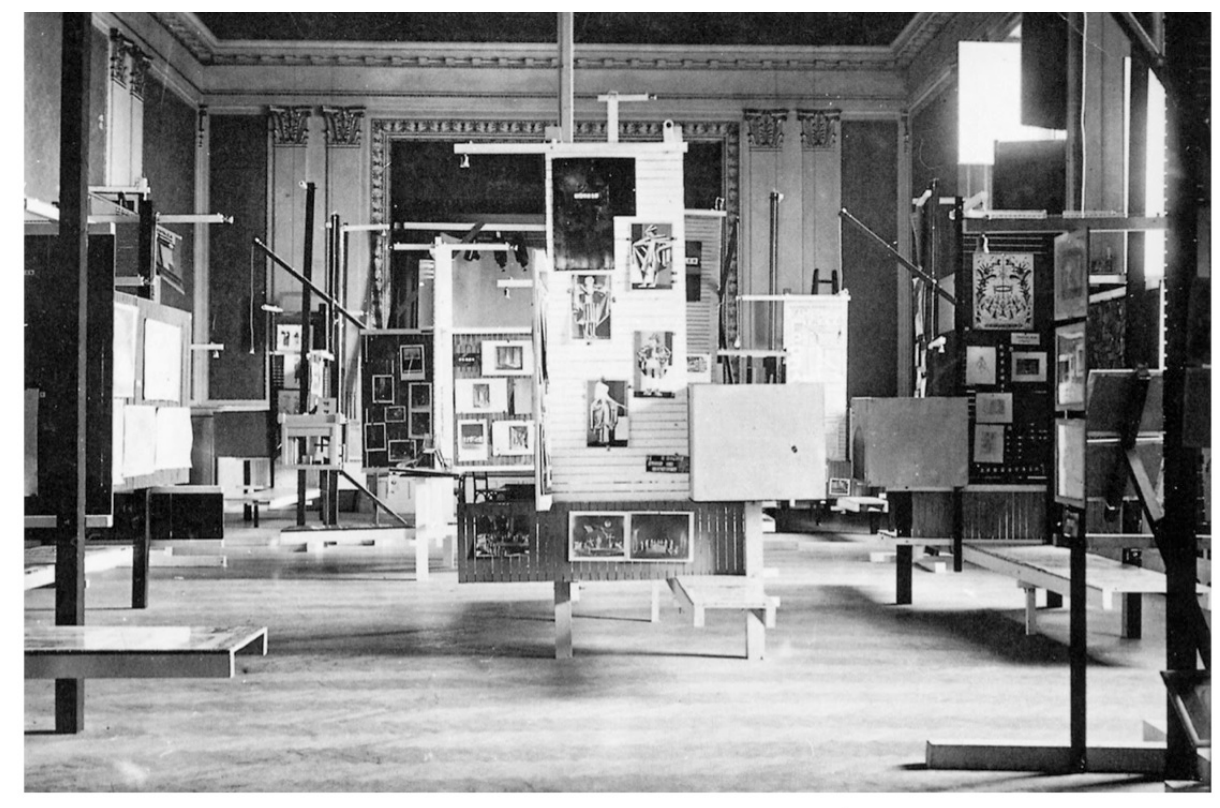


Kiesler teve a oportunidade de escrever sobre seu novo sistema na revista De Stijl, e o editor, Theo van Doesburg, que havia visitado a exposição publicou o seguinte elogio:

Enquanto viajava pela Alemanha, França, Holanda e Itália, estudei os resultados dos mais novos empreendimentos no campo da arquitetura. Fiquei completamente surpreso ao enfrentar a forma completamente nova de demonstração na Exposição Internacional de Teatro da Nova Técnica de Teatro de Viena. Em nenhuma cidade do mundo vi algo parecido. Ao contrário das exposições anteriores, nas quais objetos de arte eram pendurados um ao lado do outro sem relação, neste método de demonstração as relações mais próximas entre as diferentes obras foram estabelecidas pelo arranjo no espaço. É extremamente importante e feliz que o Festival de Teatro e Música tenha encontrado uma solução básica, prática e econômica para esse problema no novo sistema de exibição criado por Kiesler ${ }^{6}$. (DOESBURG, 1943, p.507 apud GOODMAN, 1989, p.57, tradução nossa)

Ainda para o mesmo evento, segundo Lesak (1989), Kieler executou seu projeto de Space Stage (Figura 5), um espaço para performances, constituído por uma rampa em espiral com um platô circular no segundo estágio.

No ano seguinte, convidado pelo arquiteto Josef Hoffmann (18701956) a participar da Exposition Internationale des Arts Décoratifs et Industriels Modernes (Exposição internacional de artes decorativas e industriais modernas) em Paris, Kiesler realiza Raumstadt (Cidade Espacial), seu projeto mais afinado com as regras do De Stijl, evoluído do seu próprio sistema L e T.

Fig 5. Space Stage, 1924

Fonte http://www.dreamideamachine. com/en/?p=24375

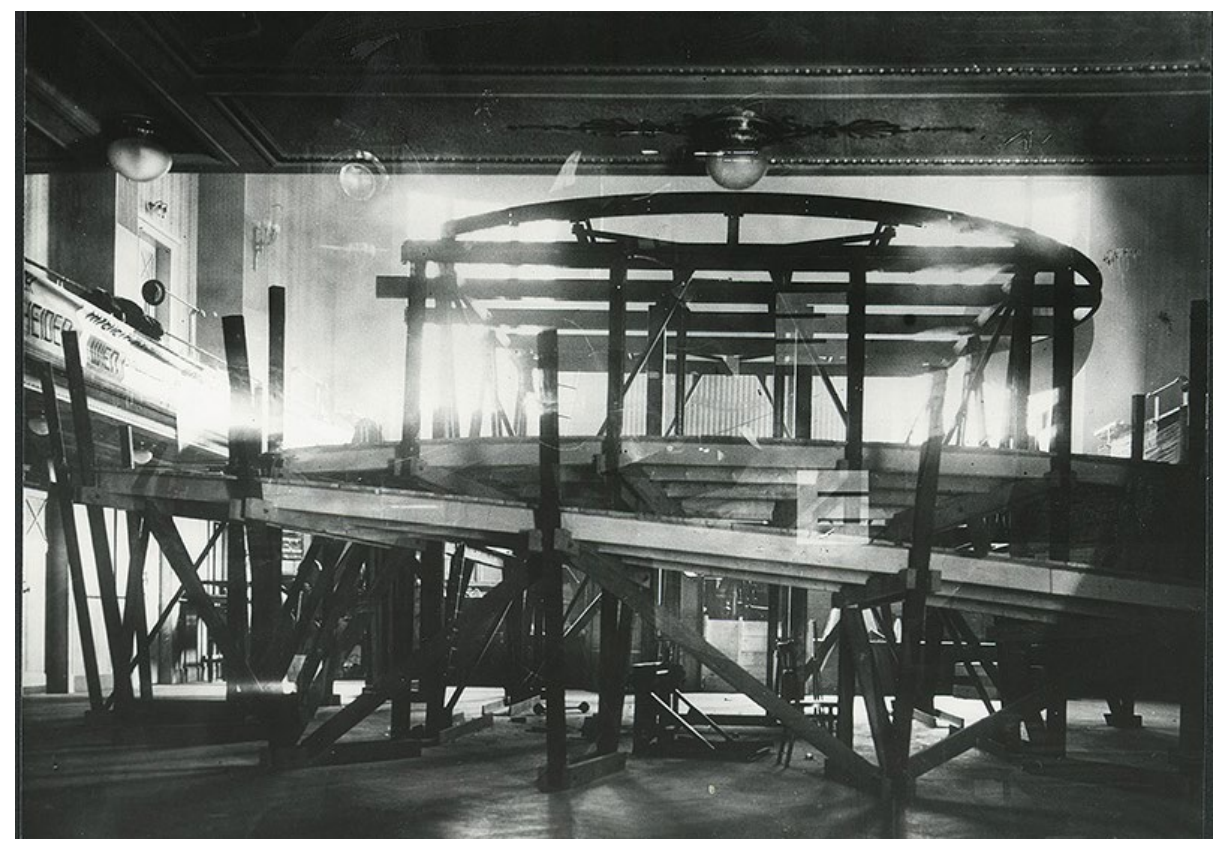

DATJournal v. 5 n.3 2020 


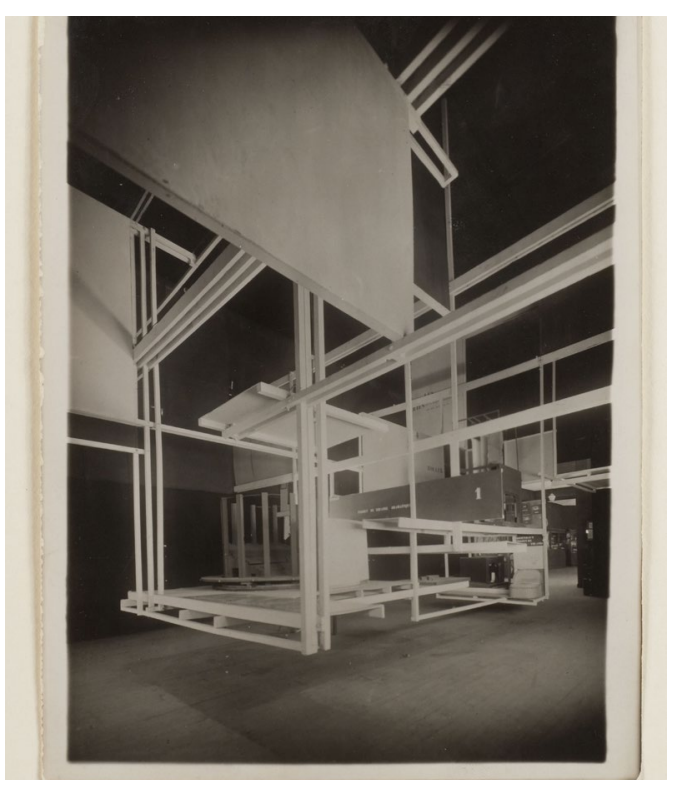

Fig 6. Cidade Espacial, Exposição internacional de artes decorativas e industriais modernas, Paris, 1925 Fonte https://www.mumok.at/ en/general-information-0
Cidade Espacial (1925) (Figura 6) era construída por painéis e vigas suspensas no espaço sem apoios, nos planos horizontal e vertical, com cores reduzidas, vermelho, branco e preto, que segundo Staniszewski (2001) possuía dupla função: ser um modelo de cidade futurista e um sistema de display para exposições.

Em manuscrito não datado citados por Goodman (1989), Kiesler escreve sobre esse período: "Foi em 1924-25, nas valsas de Viena de Strauss e em Paris da Beaux-Arts, que eliminei a separação entre piso, paredes e teto e criei pisos, paredes e teto, como um todo contínuo"s (KIESLER ${ }^{9}$ apud GOODMAN, 1989, p.57, tradução nossa).

Em 1926, Kiesler teve oportunidade de mostrar seu sistema expositivo flexível em New York, na International Theatre Exposition (Exposição Internacional de Teatro) que ocorreu no Steinway Hall. Convidado por Jane Heap (1883-1964), editora da revista de vanguarda americana The Little Review, que esteve em Paris e havia visto a sua Cidade Espacial. (LESAK, 1989)

\section{Kiesler no Novo Mundo, New York}

Para Phillips (1989a), a Exposição Internacional de Teatro de 1926, teria sido uma das primeiras oportunidades que New York teve de entrar em contato com a produção artistas como Picasso (1881-1973), El Lissitzky, Oskar Schlemmer, Enrico Prampolini (1894-1956) como designers cenográficos e as influências das estéticas europeias da primeira metade do século XX.

No final da década de 1920, Kiesler havia se mudado com sua esposa para New York, adquirido reconhecimento dos círculos de artistas, intelectuais e boêmios de Greenwich Village, mas não conseguindo sucesso financeiro, mantinha dívidas. Suas mais importantes realizações foram o Film Guild Cinema e as vitrines da loja de departamentos Saks Fifth Avenue.

Film Guild Cinema (Figura 7) abre em 1929 e sua sala de projeção abolia o tradicional proscênio e possuía chão e tetos projetados gradualmente inclinados em direção à tela, sendo possível compará-la ao interior de uma câmera fotográfica, com o característico diafragma. Buscava com a estrutura otimizar a acústica e o ângulo de visão da tela. Phillip (1989b) destaca as características do empreendimento com claras preocupações de De Stijl e dos Construtivistas. A autora ainda comenta que no projeto original de Kiesler havia dois sistemas de projeção múltipla, com tela expansível, e com mais três telas auxiliares, um sistema de iluminação que circundaria toda a sala a fim de criar uma experiência ambiental arquitetônica que envolvesse o usuário, porém os sistemas não foram realizados. 


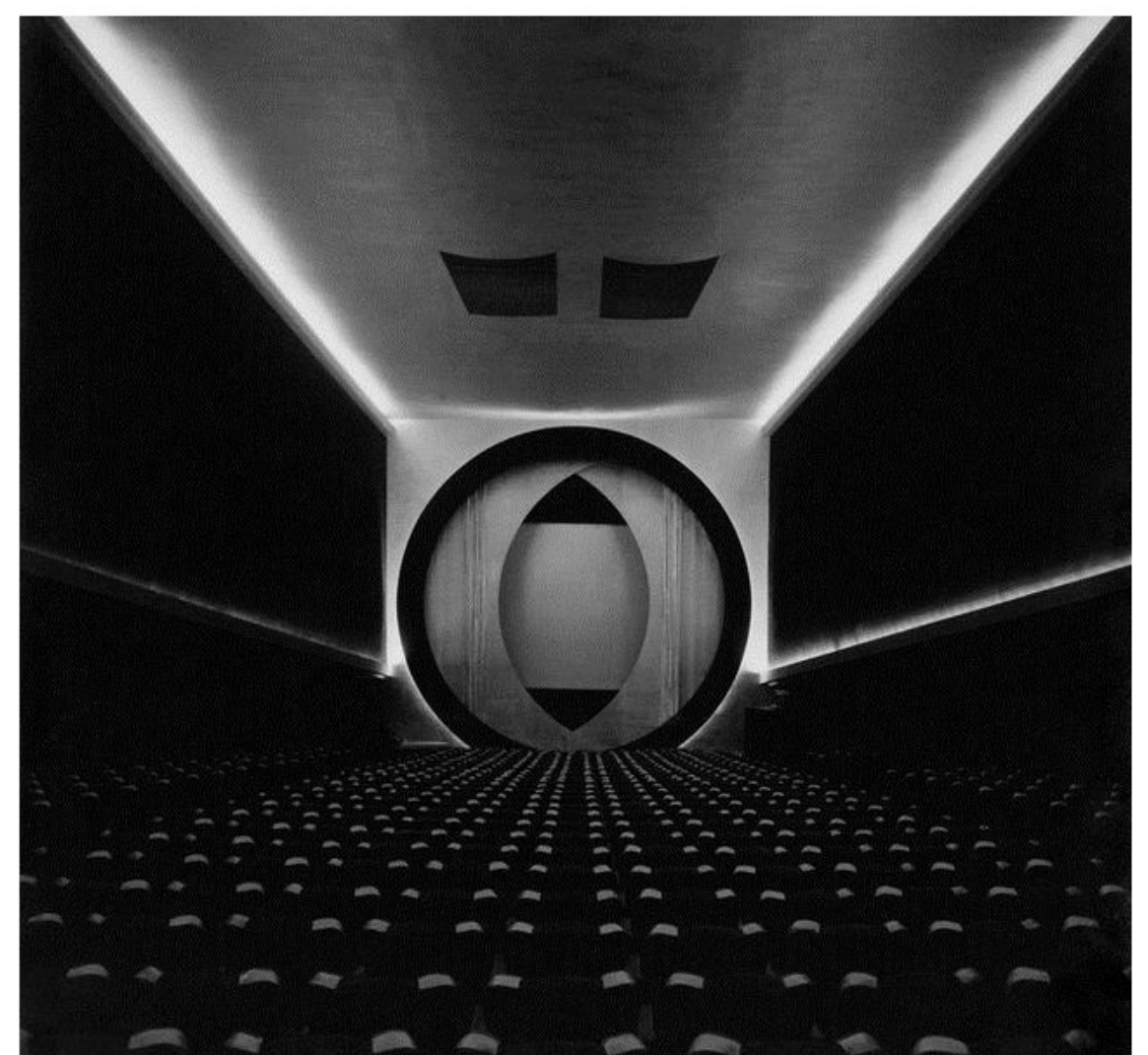

Fig 7. Film Guild Cinema, New York,1929 Fonte https://www.design-is-fine. org/post/158806418234/friedrich-kiesler-film-guild-cinema

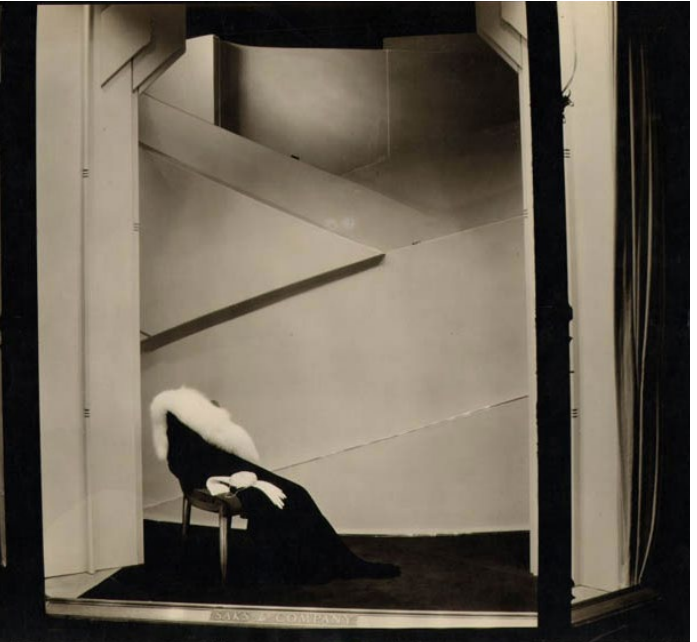

Fig 8. Vitrine para Saks Fifth Avenue, New York, 1927/28

Fonte https://www.bmiaa.com/ frederick-kiesler-architect-artist-visionary-at-martin-gropius-bau-berlin/mgb17_kiesler_ schaufenster_saks_litebox
Para Goodman (1989), ao projetar as vitrines da loja de departamento Saks Fifth Avenue, Kiesler (Figura 8 e Figura 9) esperava exercer o mesmo efeito que obras numa galeria em relação a seus públicos. Em suas composições, privilegiava arranjos assimétricos dramáticos, o mínimo de adereços por vitrine, monocromia e frequentemente o abandono dos manequins (PHILLIPS, 1989a).

Publica, em 1930, Contemporary Art Applied to the Store and Its Display, espécie de manual que descrevia os movimentos estéticos europeus da primeira metade do século XX como Cubismo, Futurismo, De Stijl e Bauhuaus, num claro esforço para divulgar os princípios do modernismo na America. No livro, segundo Phillips (1989a), Kiesler comenta que os Estados Unidos haviam na época alcançado a liderança em quase todos os campos, menos nas artes. E creditava as lojas de departamento a introdução do modernismo a população em geral. Ainda no livro, segundo Goodman (1989), o arquiteto detalhava considerações sobre arranjos compositivos para melhor expor as mercadorias, como evitar as simetrias, posicionar os produtos em alturas variáveis e considerar as proporções da moldura da vitrine. 
Foram criadas quatro áreas a pedido de Guggenheim para The Art of This Century: a galeria surrealista, a galeria de arte abstrata, a galeria cinética e uma biblioteca.

Fig 11. Galeria Surrealista, The Art of This Century

Fonte https://curatorialexperiments. wordpress.com/category/sources/

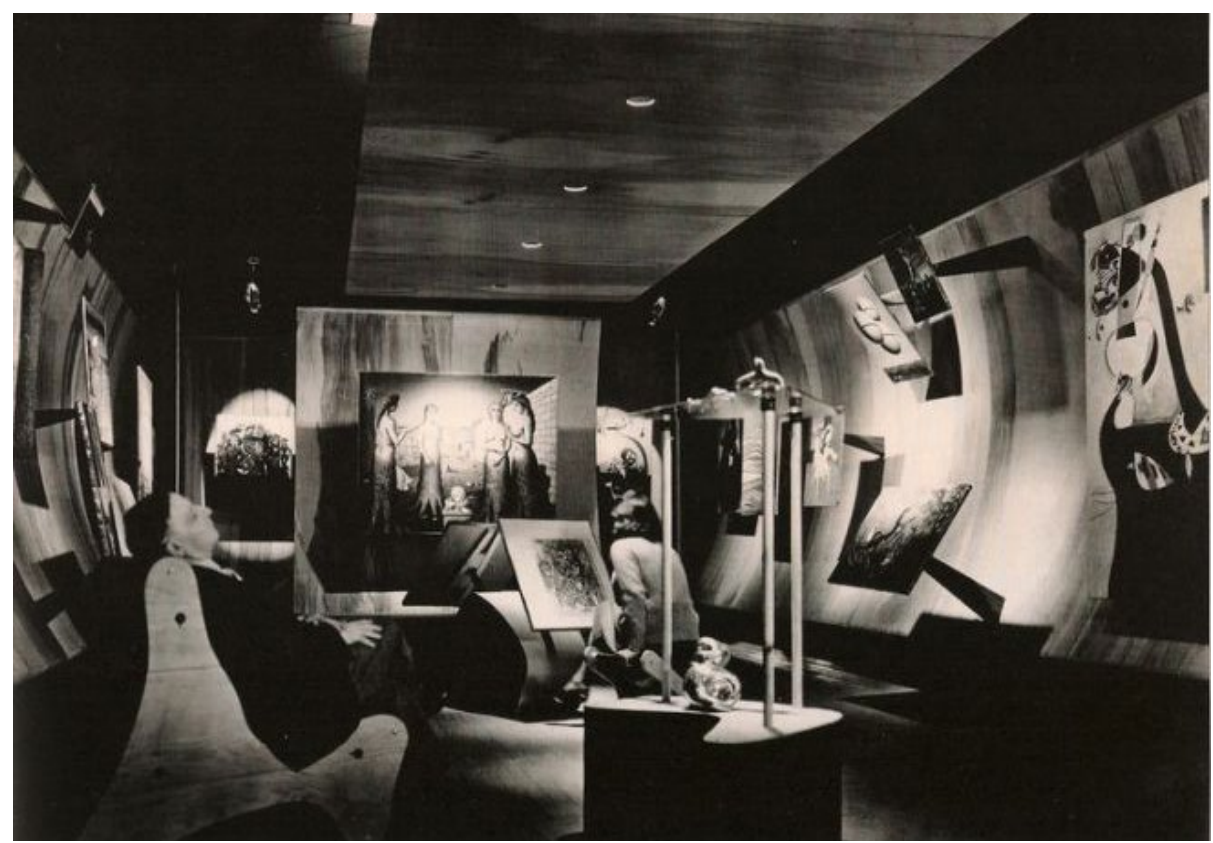

A Galeria Surrealista (Figura 11) apresentava um espaço fluido e curvilíneo graças as paredes curvadas de madeira sobre as originais. A iluminação projetada de tal maneira que destacava cada um dos lados da sala, de forma intercalada a cada dois minutos. 0 ruído de um trem periodicamente se repetia. Junto a isso pinturas suspensas nos displays flexíveis, proporcionavam uma experiência aumentada, onde visão, audição, deslocamento e toque eram estimulados. Arranjos triangulares com finas fitas de tecido, permitiam o visitante levantar e baixar as obras que pareciam manter-se suspensas no ar na Galeria Abstrata (Figura 12).

Fig 12. Galeria Abstrata,

The Art of This Century

Fonte https://www.guggenheim-venice.it/en/art/in-depth/peggy-guggenheim/about-peggy/

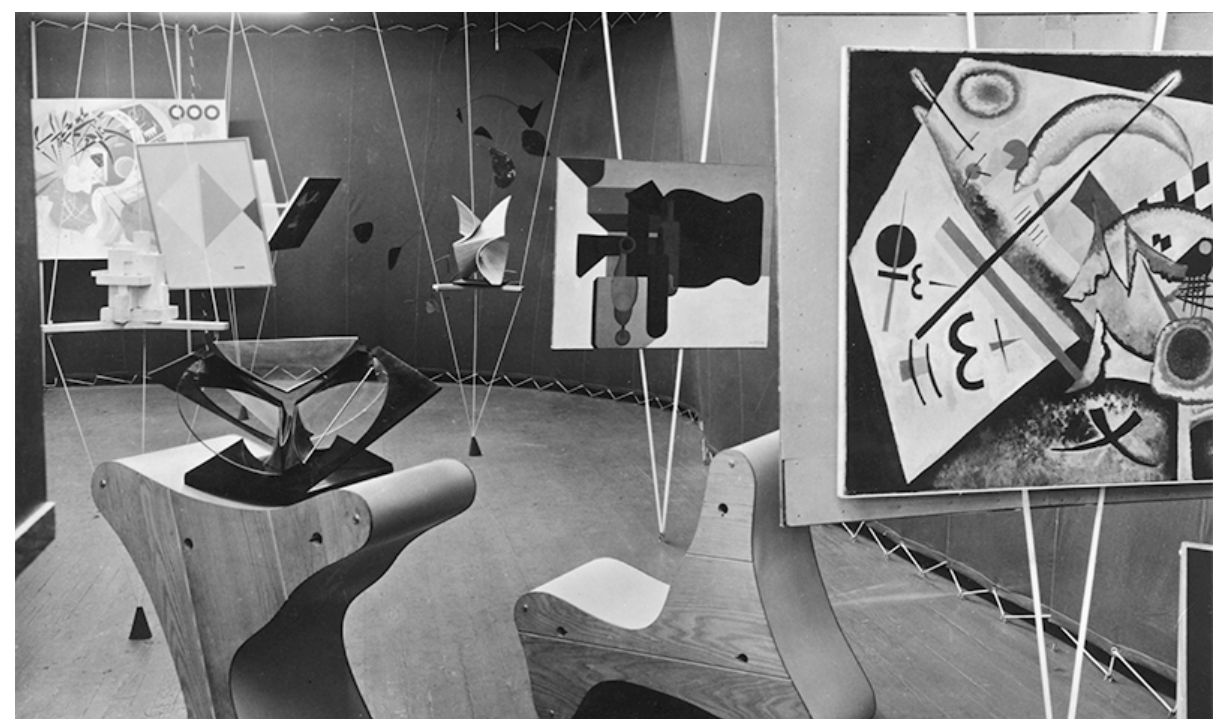


Fig 13. Mecanismo para ver "Boîte-envalise" de Marcel Duchamp, Galeria Cinética, The Art of This Century Fonte http://www.dreamideamachine. com/en/?p=24375
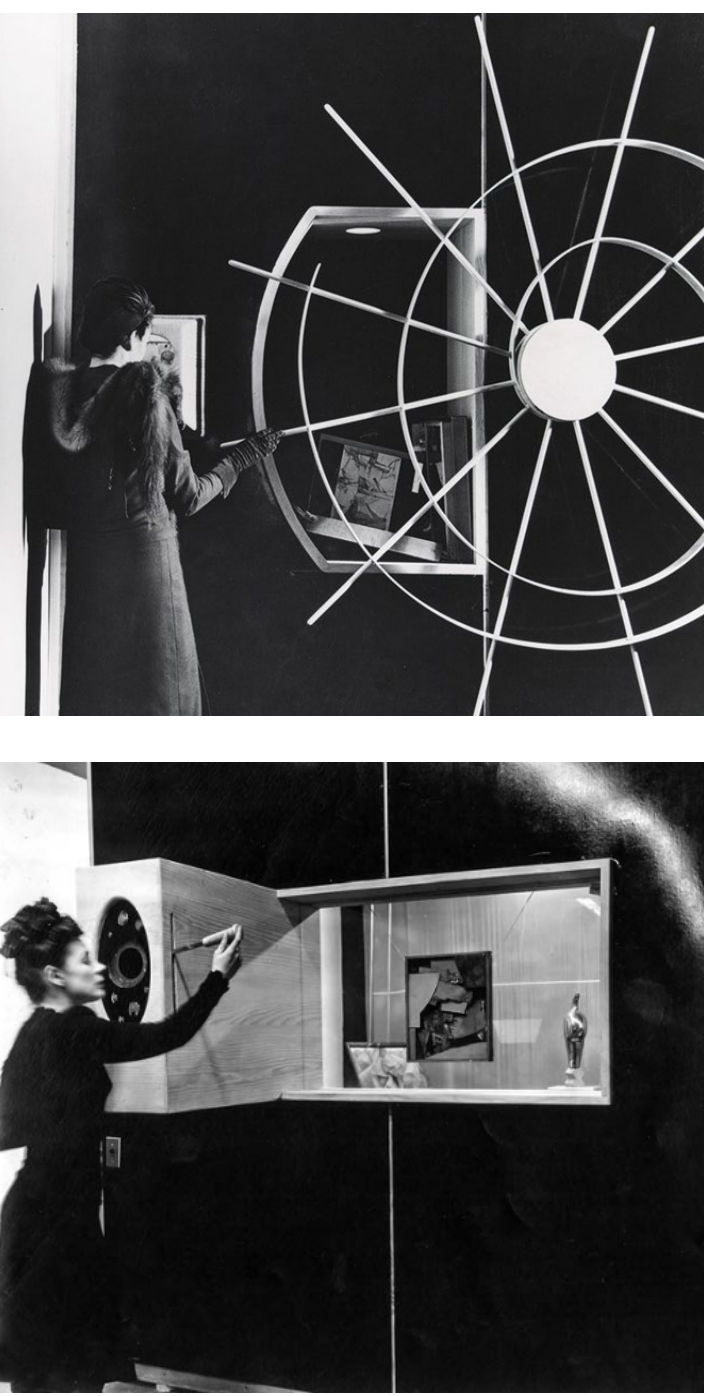

Fig 14. Caixa de Sombras, Galeria Cinética, The Art of This Century Fonte PHILLIPS, Stephen J. Elastic Architecture - Frederick Kiesler and Design Research in the First Age of Robotic Culture. The MIT Press. London. 2017. p. 178
Na Galeria Cinética trabalhos eram exibidos graças a mecanismos ativados pelo público, olhando por um olho mágico e ao rodar uma grande roda de madeira era possível ver as catorze reproduções pertencentes a La Boîte-en-valise de Marcel Duchamp (Figura 13), em outra engenhoca similar, pinturas de Paul Klee eram exibidas em sucessão uma a outra, um terceiro dispositivo era acionado por meio de uma alavanca, tratava-se de uma caixa de sombras, na qual um retrato de André Breton (1996-1966) Portrait of the Actor A.B estava instalado (Figura 14).

A chamada Biblioteca (Figura 15), era uma galeria para exposições temporárias, na qual os visitantes podiam, sentados em bancos dobráveis, estudar e manipular obras de arte que se encontravam disponibilizadas.

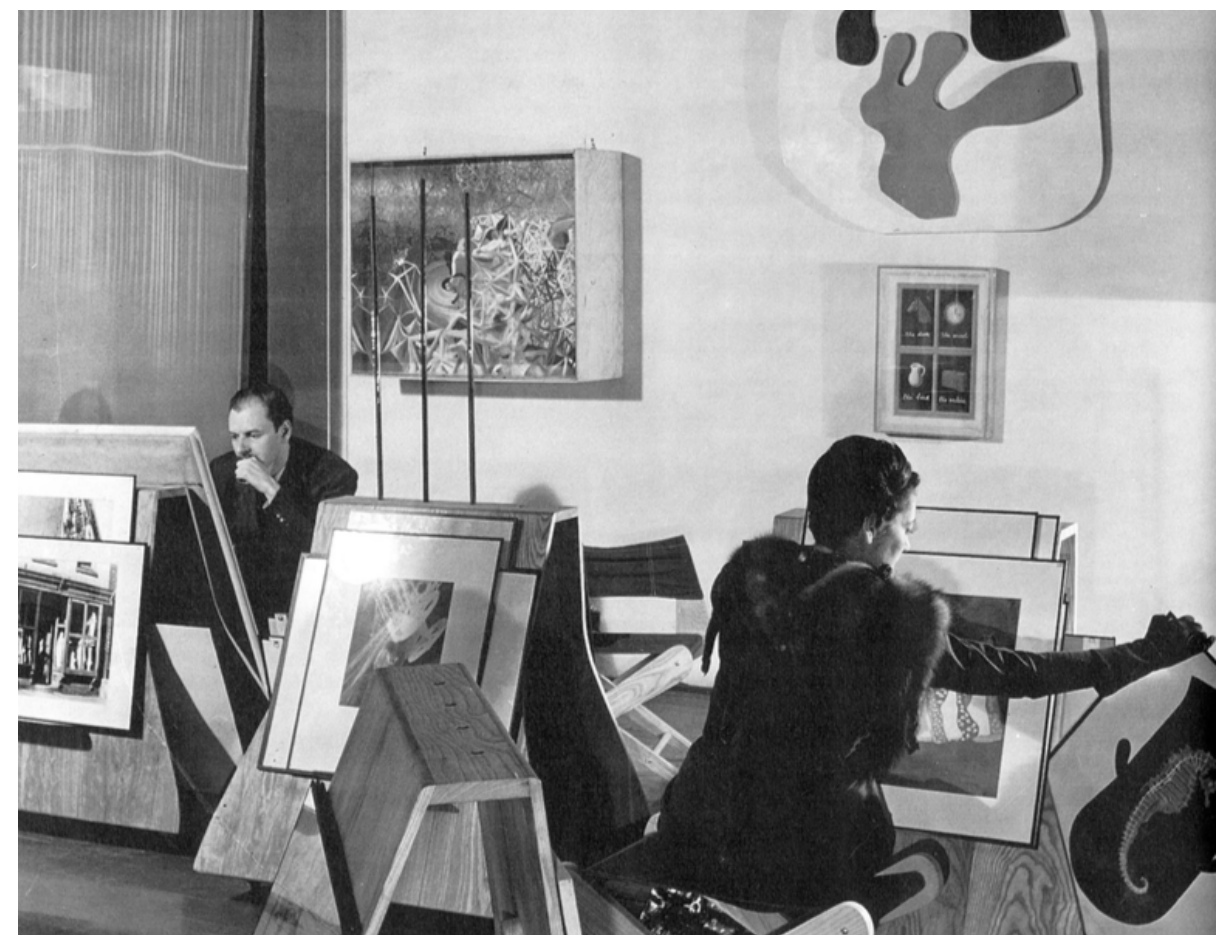

Fig 15. Biblioteca, The Art of This Century

Fonte STANISZEWSKI, Mary Anne. The power of display: a history of exhibition installations at the Museum of Modern Art. Cambridge: MIT Press, 2001. p. 10 


\section{Bloodflames e a Exposition Internacionale du Surrealisme}

Desde que fora introduzido no círculo dos surrealistas por Marcel Duchamp, Kiesler se tornou o único arquiteto reconhecido pelo grupo (PHILLIPS, 2007b). Em suas pesquisas no laboratório de design nas universidades de Columbia e Yale, junto a experiências sobre a natureza da percepção visual e como a arquitetura poderia afetá-la, eram cada vez mais frequentes, considerações sobre a mente, o inconsciente e a atividade dos sonhos. Em suas preocupações se destacavam parâmetros psicológicos e fisiológicos (PHILLIPS, 2010), acreditando que o psicológico era de maior importância e significado, pois um "visitante psicologicamente estimulado, teria sua fadiga física reduzida"13 GOODMAN, 1989, p.62).

Em 1947 Kiesler teve a oportunidade de executar dois designs de exposições: Bloodflames organizada por Nicolas Calas (1907-1988) na Galeria Hugo de New York, e a Exposition Internationale du Surrealisme na Galerie Maeght em Paris organizada por André Breton.

Bloodflames reuniu trabalhos de David Hare (1852-1917), Arshile Gorky (1904-1948), Roberto Matta (1911-2002), Isamu Noguchi (1904-1988), Jeanne Reynal (1903-1983), Gerome Kamrowski (1914-2004), Wilfredo Lam (1902-1982) e Helen Phillips (1913-1994). Na mostra Calas e Kiesler proclamavam a necessidade da integração entre pintura e arquitetura. Proclamavam no catálogo: "É preciso acabar com o divórcio entre arquitetura e pintura"14 (CALAS; KIESLER, 1947, p.16 ${ }^{15}$ apud PHILLIPS, 2007b, p.165). Desafiavam a galeria típica e o design funcional.

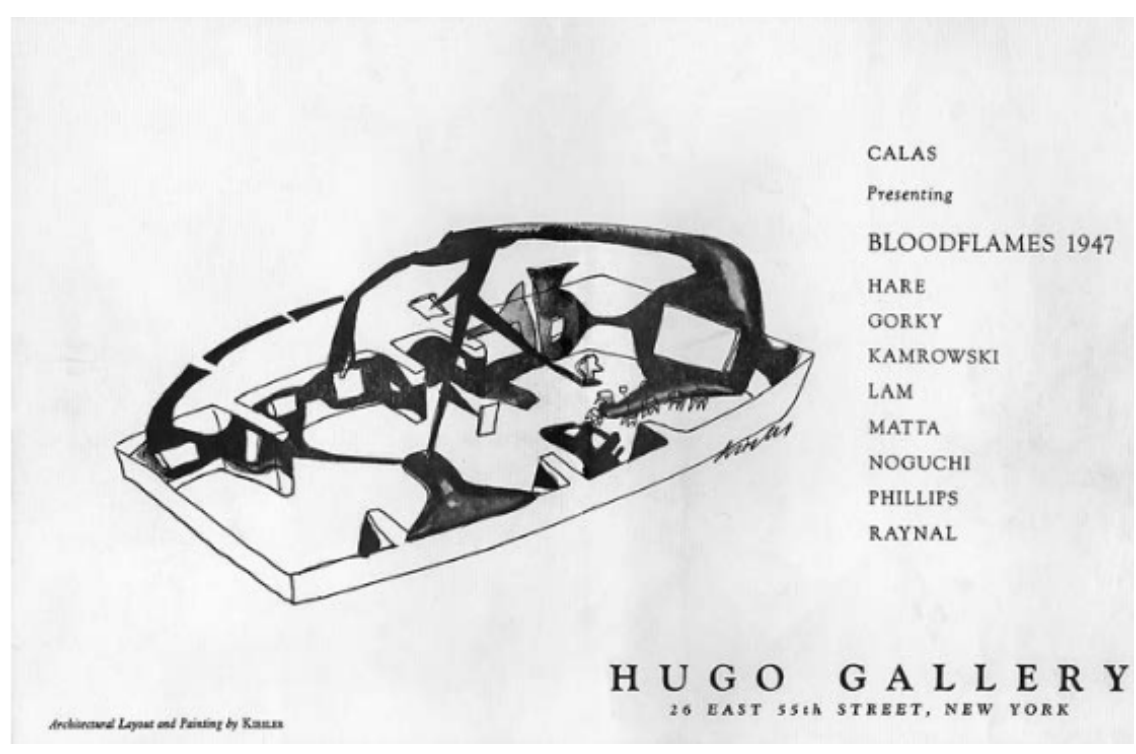

Fig 16. Capa do catálogo de Bloodflames. Fonte PHILLIPS, Stephen J. Elastic Architecture - Frederick Kiesler and Design Research in the First Age of Robotic Culture. The MIT Press. London. 2017. p.167 
Por se tratar de uma mostra temporária, baixos custos e rápida velocidade de execução deveriam ser levados em conta, junto da sua preocupação de estabelecer uma continuidade espacial. A solução foi pintar faixas coloridas ondulantes estabelecendo continuidade entre piso, teto e paredes e dispensar a construção de dispositivos (Figura 16), a forma mais barata de modificar um espaço segundo Kiesler. Em suas palavras: "As formas coloridas fornecem os meios mais simples, baratos e rápidos para transformar uma sala: o homem de baixa renda pode pelo menos ficar bêbado com um pote de tinta." ${ }^{16}$ (KIESLER ${ }^{17}$ apud GOODMAN, 1989, p.69, tradução nossa)

Os trabalhos foram dispostos no chão, tortos nas paredes parecendo

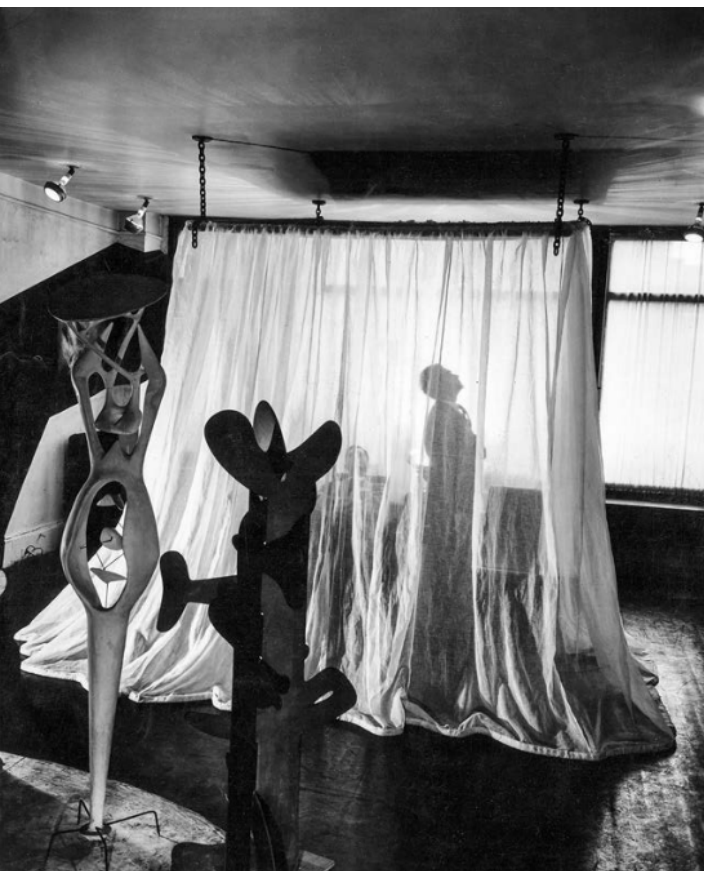

Fig 18. Espaço com cortinas para A presença Eterna (1944) de Wilfredo Lam Fonte PHILLIPS, Stephen J. Elastic Architecture - Frederick Kiesler and Design Research in the First Age of Robotic Culture. The MIT Press. London. 2017. p.186 desequilibrados ou mesmo suspensos no teto (Figura 17). Também fez uso de tecidos criando uma espécie de tenda onde, em seu interior, encontrava-se a pintura A presença Eterna (1944) de Wilfredo Lam de temática sexual (GOODMAN, 1989), uma espécie, para Phillips (2007b), de peep show. Havia também a cadeira, na qual Kiesler induzia o espectador a sentar-se para contemplar a imagem no teto (Figura 18 e Figura 19). Olhos eram conduzidos pelo espaço por uma faixa de Moebios, uma faixa sem fim contorcida em si mesma, levando os corpos a se inclinarem e se reposicionarem continuamente em muitas posições. Bloodflames "realizou a visão de Kiesler de correlacionar uma organização contínua de peças desconectadas em um espaço elástico contínuo, curando a divisão que ele acreditava estar entre visão e fato (sonhos e realidade)." ${ }^{18}$ (p.166)

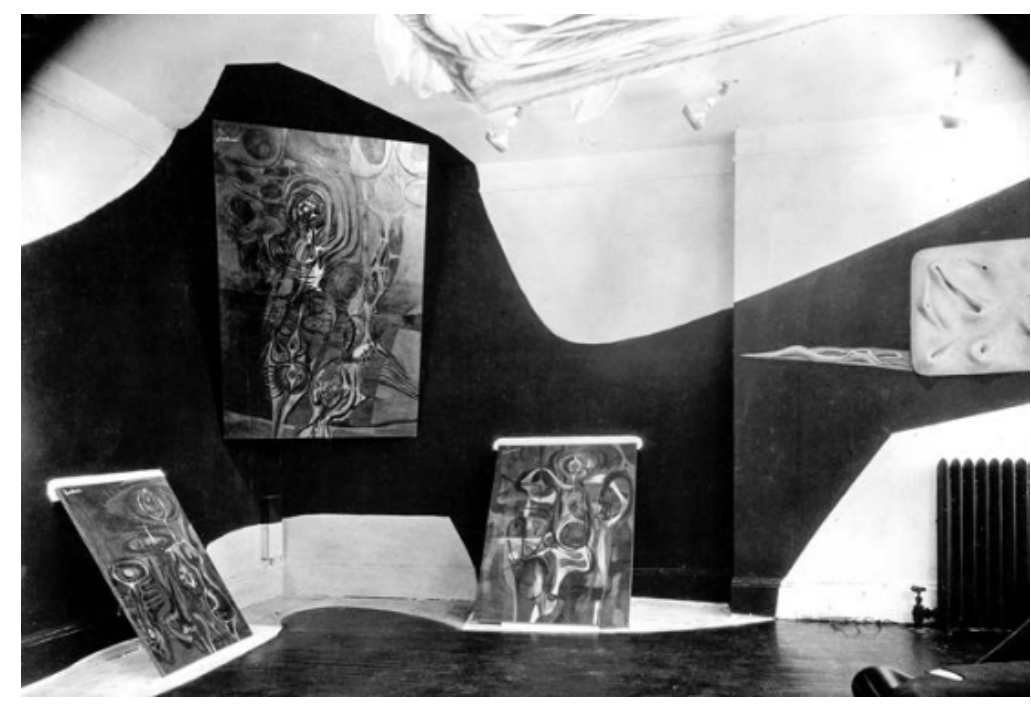

Fig 17. Exposição “Bloodflames”, Nova York, 1947 Fonte PHILLIPS, Stephen J. Elastic Architecture - Frederick Kiesler and Design Research in the First Age of Robotic Culture. The MIT Press. London. 2017. p.189
Fig 19. A presença Eterna (1944) de Wilfredo Lam vista no teto Fonte PHILLIPS, Stephen J. Elastic Architecture - Frederick Kiesler and Design Research in the First Age of Robotic Culture. The MIT Press. London. 2017. p.188 
Fig 20. Salão das Superstições na "Exposição Internacional do Surrealismo", Galeria Maeght, Paris, 1947 Fonte KRAUS, Eva Kraus. Breton Duchamp Kiesler - Surreal Space. Austrian Frederick and Lillian Kiesler Private Foundation. Viena. 2013. p. 14
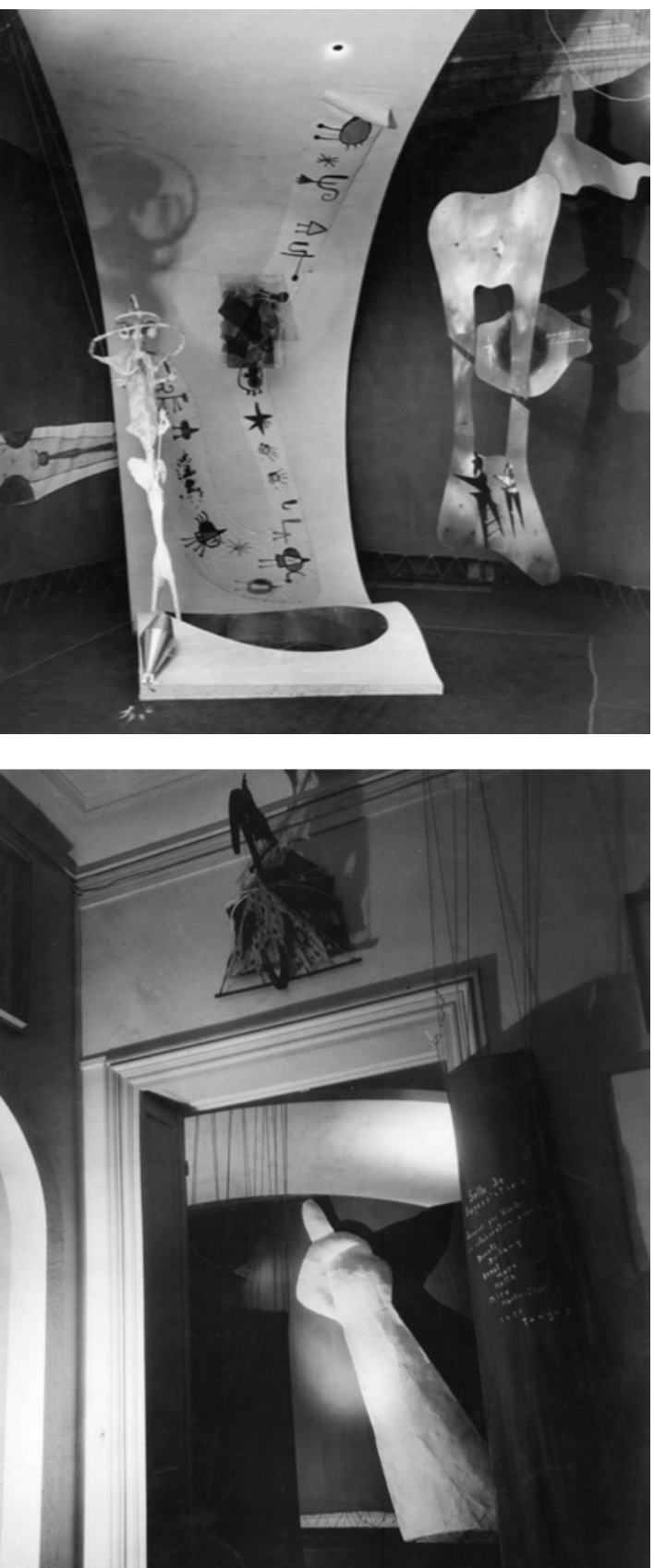

Fig 21. Entrada para o Salão de Superstições com a Figura Antitabu Fonte KRAUS, Eva Kraus.
A convite de André Breton, Kiesler viaja a Paris no mesmo ano para projetar a instalação da Exposition Internationale du Surrealisme na Galerie Maeght. A organização geral era de Breton, mas Duchamp havia feito contribuições, sendo a proposta do labirinto e o Salão de Superstições (Figura 20) suas ideias (GOODMAN, 1989). O principal objetivo de Kiesler era de que "deveria ter artistas e escultores fazendo novas obras para ser integrado com a nova arquitetura, alinhada e unida por uma visão de poeta"19 (KIESLER $^{20}$ apud PHILLPS, 2007b, p. 193, tradução nossa). Responsável pela Sala de Superstições coordenou trabalhos de Joan Miró (1893-1983), Duchamp, Roberto da Matta, Yves Tanguy (1900-1955), Max Ernst (1891-1976), David Hare e Maria Martins (1894-1973), que trabalharam de forma livre a partir de uma estrutura conceitual a partir de uma vaga noção de superstição.

Cortinas curvas funcionavam como paredes, e em alguns pontos dessas, Kiesler criou orifícios para que ao olhar através deles algumas obras fossem reveladas, como no caso da pintura de Max Ernst. Kiesler contribuiu com duas obras: a Figura Antitabu, uma grande mão de gesso com dedo levantado, posicionado logo na entrada (figura 21) a receber os visitantes e Totem para todas as religiões, estrutura construída com troncos e toras.

O Salão de Superstições deveria obrigatoriamente ser o primeiro espaço a ser visitado pelo público, uma espécie de pré-requisito a ser superado para seguir um esquema pensado por Breton de etapas sucessivas de uma iniciação. Linhas curvas unificavam visualmente todo os espaços (GOODMAN, 1989).

\section{Considerações Finais}

Com sua abordagem diversa e experimental Kiesler promoveu propostas expositivas pouco ortodoxas. Suas composições incialmente influenciadas pelos princípios do De Stjil, acabam adquirindo com o passar dos anos um novo vocabulário e se tornam mais biomórficas. O que sempre se manteve foi a preocupação de criar sistemas ambientais flexíveis com estruturas adaptáveis às necessidades e desejos do usuário.

Seus projetos expositivos propunham espaços dinâmicos e interativos, nos quais de forma ativa e criativa o visitante criava significados a partir de suas percepções e experiências. Kieser não projetava exibições para um homem universal e estático, o homem utópico do modernismo funcionalista, o qual habitava as construções retilíneas da máquina de morar. Seus designs expositivos elásticos eram pensados para um indivíduo em movimento e evolução no tempo e espaço. Suas exposições eram concebidas para habitar e performar. 
1 the dynamics of continual interaction between man and his natural and technological environments

2 KIESLER, Frederick. On Correalim and Biotechique: definition and test of a new approach to building design. Architectural Record,86. September 1939. p. 61

3 The traditional art object, be it a painting, a sculpture, or a piece of architecture, is no longer seen as an isolated entity but must be considered within the context of this expanding environment. The environment becomes equally as important as the object, if not more so, because the object breathes into the surrounding and also inhales the realities of the environment no matter in what space, close or wide apart, open air or indoor

4 KIESLER, F. Second Manifesto of Correalim. Art International 9, n2. march 1965. p. 16

5 Kiesler's freestanding structures brought the works of art into the space of the viewer and created what Kiesler called a "varied transparency. 6 While traveling through Germany, France, Holland, and Italy, I studied the results of the newest endeavors in the field of architecture. I was completely taken by surprise when I faced the completely new form of demonstration at the International Theater Exposition of New Theatre Technique at Vienna. In no city in the world have I seen anything similar to it. In contrast to previous exhibitions in which art objects were hung next to one another without relation, in this method of demonstration the closest relations between the different works were established by their arrangement in space. It is extremely important and fortunate that the Theater and Music Festival has found a basic, practical, and economical solution to this problem in the new exhibition system created by Kiesler.

7 DOESBURG, Theo van Doesburg, quoted in New Display Techniques for 'Art of This Century', Designed by Frederick Kiesler. Architectural Forum, 78. February 1943. p. 50.

8 It was in 1924-25, in the Vienna of Strauss waltzes, and in the Paris of the Beaux-Arts, that I eliminated the separation between floor, walls, and ceiling, and created floors, walls, and ceiling, as a continuous whole 9 KIESLER, Frederick. Undated. manuscriptin Kiesler Estate Archives. untitled.

10 crucial in keeping modernism alive in America, by providing continuous access to the most advanced ideas from Europe.

11 an intermediary for European Constructivism in America.

12 Today the framed painting on the wall has become a decorative cipher without life and meaning, or else, to the more susceptible observer, an object of interest existing in a world distinct from his. Its frame is at once symbol and agent of an artificial duality of "vision" and "reality," or "image" and "environment," a plastic barrier across which man looks from the world he inhabits to the alien world in which the work of art has its being. That barrier must be dissolved: the frame, today reduced to an arbitrary rigidity, must regain its architectural spatial significance.

13 the visitor were psychologically stimulated, physical fatigue would be lessened.

14 An end must be brought to the divorce between architecture and painting

15 Nicolas Calas and Frederick Kiesler, Bloodflames 1947, exh. cat. (New York: Hugo Gallery, 1941), p.16

16 Color-Forms provide the simplest, least expensive and most rapid means for transforming a room: The man of low income can at least get drunk on a pot of paint.

17 KIESLER, Frederick. Undated. manuscriptin Kiesler Estate Archives. untitled.

18 realized Kiesler's vision of correlating a seamless organization of disconnected parts into one continuous elastic space, healing the split he believed stood between vision and fact (dreams and reality).

19 was to have artists and sculptors make new works to be integrated with new architecture, lined and bound together by a poet's vision 20 KIESLER, Frederick. Art: Or the Teaching of Resistance, Commencement address give by Frederick Kiesler at the Art Institute of Chicago, June 12, 1959, and presented at the "Art and Education" conference, University of Michigan, October 18, 1958, 6, Txt 01 Man/Typ Various A, Folder Art or the Teaching of Resistance lecture Materials, Austrian Frederick and Lillian Kiesler Private Foundation Archive, Vienna.

DATJournal v. 5 n.3 2020 


\section{Referências}

BAMBOZZI, L. Da curadoria de artista a alguma outra coisa. DAT Journal, v. 4, n. 2, p. 3-21, 5 ago. 2019.

BOGNER, Dieter. Kiesler and European Avant-garde in PHILLIPS, Lisa. Frederick Kiesler. Catálogo da exposição. Whitney Museum of American Art, New York. 1989 GOODMAN, Cyntihia. The Art of Revolutionary Display Techniques in PHILLIPS, Lisa. Frederick Kiesler. Catálogo da exposição. Whitney Museum of American Art, New York. 1989 LESAK, Barbara. Visionary of the European Theater in PHILLIPS, Lisa. Frederick Kiesler. Catálogo da exposição. Whitney Museum of American Art, New York. 1989 PHILLIPS, Lisa. Archeitct of Endless Innovation in PHILLIPS, Lisa. Frederick Kiesler. Catálogo da exposição. Whitney Museum of American Art, New York. 1989a PHILLIPS, Lisa. Environmental Artist in PHILLIPS, Lisa. Frederick Kiesler. Catálogo da exposição. Whitney Museum of American Art, New York. 1989b PHILLIPS, Stephen J. Elastic Architecture - Frederick Kiesler and Design Research in the First Age of Robotic Culture. The MIT Press. London. 2017

PHILLIPS, Stephen J. Toward a Research Practice: Frederick Kiesler's Design-Correlation Laboratory. Grey Room. N. 38 | Winter 2010 p.90-120

STANISZEWSKI, Mary Anne. The power of display: a history of exhibition installations at the Museum of Modern Art. Cambridge: MIT Press, 2001 